\title{
Coherent Multiple Scattering Effect in DIS *
}

\author{
Xiaofeng Guo and Jun Li \\ Department of Physics and Astronomy, Iowa State University \\ Ames, Iowa 50011, U.S.A.
}

\begin{abstract}
We present a QCD calculation of the coherent multiple scattering effect on single hadron production in semi-inclusive lepton-nucleus deep inelastic scattering (DIS). We show that the destructive interference of coherent multiple scattering leads to suppression in hadron productions. The suppression is similar to the expectations of energy loss calculations. We compare our calculation with experimental data.
\end{abstract}

Key words: Multiple scattering, energy loss, deep inelastic scattering PACS: $12.38 . \mathrm{Bx} 13.85 . \mathrm{Ni} 24.85 .+\mathrm{p} 25.75 .-\mathrm{q}$

1. Introduction The strong suppression of high transverse momentum hadron in relativistic heavy ion collisons was considered to be an evidence for the QCD quark-gluon plasma [1]. The suppression was believed to be the result of medium induced radiative energy loss of high energy partons [2]. However, recent data indicate that heavy quarks would have to lose the same amount of energy as that of a light quark if the radiative energy loss is the only source of the suppression [3]. On the other hand, we expect heavy quarks to lose much less energy than a light quark because of its mass [4]. In this talk, we present a new source of suppresion from the destructive multiple scattering in a nuclear medium [5]. We calculated the single hadron production in DIS in perturbative QCD, and found that the coherent interference results into a suppression in the hadron production rate.

2. Single hard scattering We consider the production of a single hadron of momentum $p_{h}$ in lepton-nucleus deep inelastic scattering (DIS), $e\left(k_{1}\right)+A(p) \longrightarrow$ $e\left(k_{2}\right)+h\left(p_{h}\right)+X$, with the target atomic weight $A$ and average nucleon momentum $p=P_{A} / A$. The semi-inclusive DIS cross section,

$$
\frac{d \sigma_{e A \rightarrow e h X}}{d x_{B} d Q^{2} d z_{h}}=\frac{1}{8 \pi} \frac{e^{4}}{x_{B}^{2} s^{2} Q^{2}} L^{\mu \nu}\left(k_{1}, k_{2}\right) \frac{d W_{\mu \nu}^{A}}{d z_{h}},
$$

ऋ Supported in part by the National Science Fundation, grant PHY-0340729. 
where $s=\left(p+k_{1}\right)^{2}$ is the total invariant mass of the lepton-nucleon system, and $z_{h} \equiv p \cdot p_{h} / p \cdot q=2 x_{B} p \cdot p_{h} / Q^{2}$ is the fraction of the photon momentum carried by the observed hadron. The Bjorken $x_{B}=Q^{2} /(2 p \cdot q)$ with $Q^{2}=-q^{2}$. The leptonic tensor $L^{\mu \nu}\left(k_{1}, k_{2}\right)=\operatorname{Tr}\left(\gamma \cdot k_{1} \gamma^{\mu} \gamma \cdot k_{2} \gamma^{\nu}\right) / 2$. For single scattering at the lowest order, the hadronic tensor can be factorized as

$$
\frac{d W_{\mu \nu}^{A}}{d z_{h}}=\frac{1}{2} e_{\mu \nu}^{T} \sum_{q} e_{q}^{2} A \phi_{q}^{A}\left(x_{B}, Q^{2}\right) D_{q}\left(z_{h}, Q^{2}\right)
$$

where $e_{\mu \nu}^{T}=\left[p_{\mu} q_{\nu}+q_{\mu} p_{\nu}\right] / p \cdot q+p_{\mu} p_{\nu}\left(2 x_{B} / p \cdot q\right)-g_{\mu \nu}, \phi_{q}^{A}\left(x_{B}, Q^{2}\right)$ is nuclear quark distribution per nucleon of flavor $q$, and $D_{q}\left(z_{h}, Q^{2}\right)$ is quark-to-hadron fragmentation function. The nuclear dependence of Eq. (2) and Eq. (1) is limited to the nuclear dependence of $\phi_{q}^{A}\left(x_{B}, Q^{2}\right)$.

3. Coherent multiple scattering Multiple scattering introduces the medium size enhanced, or the $A^{1 / 3}$-type, nuclear effects. We adopt the leading pole approaximation to extract the leading contributions in powers of $A^{1 / 3}[6]$.

For double scattering at lowest order in $\alpha_{s}$, we only need to consider two diagrams in Fig. 1. When $x_{B} \geq x_{c} \sim 0.1$ [7], we consider coherent multiple scattering effects between the scattered quark and the partons of the nuclear target at the same impact parameter in the Breit frame and obtain [5]

$$
\begin{aligned}
\frac{d W_{\mu \nu}^{(1)}}{d z_{h}} & =\frac{1}{2} e_{\mu \nu}^{T} \sum_{q} e_{q}^{2}\left[\frac{4 \pi^{2} \alpha_{s}}{3}\right] \frac{z_{h}}{Q^{2}} T_{q g}^{A}\left(x_{B}, Q^{2}\right) \frac{d D\left(z_{h}, Q^{2}\right)}{d z_{h}} \\
& \approx \frac{1}{2} e_{\mu \nu}^{T} \sum_{q} e_{q}^{2}\left(A \phi_{q}^{A}\left(x_{B}, Q^{2}\right)\right)\left[\frac{z_{h} \xi^{2}\left(A^{1 / 3}-1\right)}{Q^{2}}\right] \frac{d D\left(z_{h}, Q^{2}\right)}{d z_{h}}
\end{aligned}
$$

where $\sum_{q}$ runs over all quark and antiquark flavors. The twist-4 quark-gluon correlation function, $T_{q g}^{A}\left(x_{B}, Q^{2}\right)$, is defined in Ref. [6]. In deriving Eq. (4), we assumed that a large nucleus is made of color neutral nucleons with a constant density. The quantity $\xi^{2}$ defined in Ref. [7] is proportional to a matrix element of a gluon density operator, and represents a characteristic scale of quark interaction with nuclear medium. If $x_{B}<x_{c}$, the hadronic tensor in Eqs. (3) and (4) has an additional term proportional to $x_{B} \frac{d}{d x_{B}} \phi_{q}\left(x_{B}, Q^{2}\right)[5]$.

For $x_{B} \geq x_{c}$, we generalized our result in Eq. (4) to $n$ scattering [5]

$$
\frac{d W_{\mu \nu}^{(n)}}{d z_{h}} \approx \frac{1}{2} e_{\mu \nu}^{T} \sum_{q} e_{q}^{2}\left(A \phi_{q}^{A}\left(x_{B}, Q^{2}\right)\right) \frac{1}{n !}\left[\frac{z_{h} \xi^{2}\left(A^{1 / 3}-1\right)}{Q^{2}}\right]^{n} \frac{d^{n} D\left(z_{h}, Q^{2}\right)}{d z_{h}^{n}}(5)
$$

We sum the $A^{1 / 3}$-type contributions in Eq. (5) to $N$ scatterings, and take $N \approx \infty$ because the effective value of $\xi^{2}$ is relatively small. We obtain 


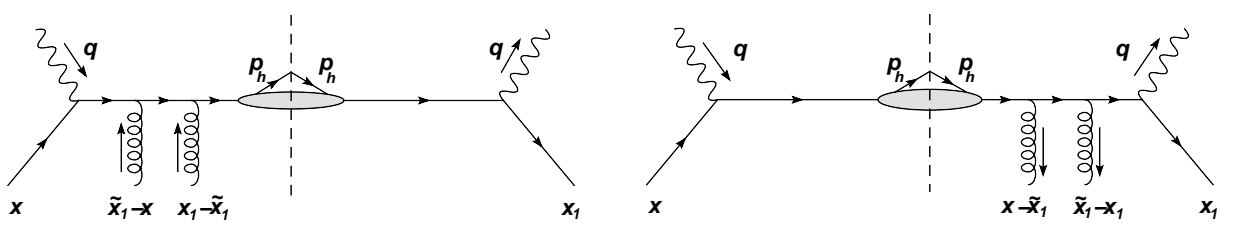

(a)

(b)

Fig. 1. Double scattering diagrams that contribute to the production rate of single hadron in DIS.

$$
\begin{aligned}
\frac{d W_{\mu \nu}^{A}}{d z_{h}} & \approx \frac{1}{2} e_{\mu \nu}^{T} \sum_{q} e_{q}^{2}\left(A \phi_{q}^{A}\left(x_{B}, Q^{2}\right)\right) \sum_{n=0}^{N} \frac{1}{n !}\left[\frac{z_{h} \xi^{2}\left(A^{1 / 3}-1\right)}{Q^{2}}\right]^{n} \frac{d^{n} D\left(z_{h}, Q^{2}\right)}{d z_{h}^{n}} \\
& \approx A \frac{1}{2} e_{\mu \nu}^{T} \sum_{q} e_{q}^{2} \phi_{q}^{A}\left(x_{B}, Q^{2}\right) D_{q}\left(z_{h}+\frac{z_{h} \xi^{2}\left(A^{1 / 3}-1\right)}{Q^{2}}, Q^{2}\right)
\end{aligned}
$$

Eq. (6) is our main result. The net effect of coherent multiple scattering of a propagating quark in the medium, is equivalent to a shift, $\Delta z$, in the variable $z_{h}$ of the quark fragmentation function $D_{q}\left(z_{h}, Q^{2}\right)$ with $\Delta z=z_{h} \xi^{2}\left(A^{1 / 3}-1\right) / Q^{2}$, which leads to a suppression of the production rate [5].

4. Comparison with HERMES data We compare our result with HERMES data [8] in Fig. 2. $R_{M}$ is the ratio of hadron production rate rate per DIS event for a nuclear target $A, R^{A}$, to that of a deuterium target $D, R^{D}$ [5],

$$
R_{M} \equiv \frac{R^{A}}{R^{D}} \approx \frac{\sum_{q} e_{q}^{2} \phi_{q}^{A}\left(x_{B}, Q^{2}\right) D_{q}\left(z_{h}+\Delta z, Q^{2}\right)}{\sum_{q} e_{q}^{2} \phi_{q}^{D}\left(x_{B}, Q^{2}\right) D_{q}\left(z_{h}, Q^{2}\right)} \frac{\sum_{q} e_{q}^{2} \phi_{q}^{D}\left(x_{B}, Q^{2}\right)}{\sum_{q} e_{q}^{2} \phi_{q}^{A}\left(x_{B}, Q^{2}\right)}
$$

The dashed lines are from Eq. (7) and the dot-dashed lines includes only the double scattering. At large $z_{h}$, resummed theory curves are much steeper than the data. This is because the formation time for the hadron or corresponding pre-hadron state is much shorter at large $z_{h}$, and therefore, the number of scattering for the propagating parton at large $z$, or the shift, should be reduced [5]. Since the hadron formation time is proportional to $\left(1-z_{h}\right)$ [9], we modify $\Delta z$ by multiplying a factor $\left(1-z_{h}\right)$ and produce the solid lines in Fig. 2.

The suppression from the coherent multiple scattering, calculated here, complements the suppression from medium-induced radiation [10], which should give additional suppression and bring down the curves in Fig. 2 [5].

5. Summary and outlook We showed that the coherent multiple scattering of a propagating quark in the medium, without the induced radiation, can change the quark fragmentation or hadron's production rate. The net effect of leading power contributions in medium length is equivalent to a shift in the fragmentation function's $z_{h} \rightarrow z_{h}+\Delta z$, and at the lowest order, $\Delta z$ is given by an universal matrix element. Our result is complementary to the energy loss of induced radiation. However, beyond the leading order, the separation 

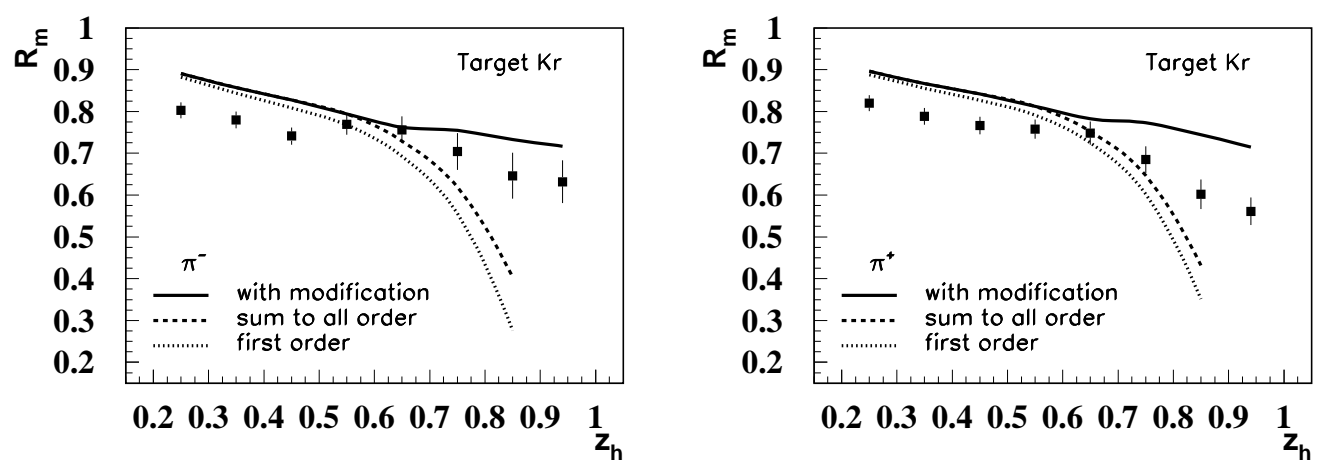

Fig. 2. Suppression from multiple scattering compared with HERMES data [8].

of the collisional energy loss and that of induced radiation will depend on the factorization scheme and is not unique, and needs further study. Our approach can be generalized to hadron productions in $p+A$ and $A+A$ collisions.

\section{References}

[1] S. S. Adler et al. [PHENIX Collaboration], Phys. Rev. Lett. 91, 072301 (2003); and references therein.

[2] M. Gyulassy and X. N. Wang, Nucl. Phys. B 420, 583 (1994); X. N. Wang, M. Gyulassy and M. Plumer, Phys. Rev. D 51, 3436 (1995); R. Baier, Y. L. Dokshitzer, A. H. Mueller, S. Peigne and D. Schiff, Nucl. Phys. B 484, 265 (1997); B. G. Zakharov, JETP Lett. 63, 952 (1996); JETP Lett. 65, 615 (1997); U. A. Wiedemann, Nucl. Phys. B 588, 303 (2000)

[3] S. S. Adler et al. [PHENIX Collaboration], Phys. Rev. Lett. 96, 032301 (2006); J. Bielcik [STAR Collaboration], Nucl. Phys. A 774, 697 (2006)

[4] Y. L. Dokshitzer and D. E. Kharzeev, Phys. Lett. B 519, 199 (2001)

[5] X. F. Guo, and J. Li, in preparation.

[6] M. Luo, J.-W. Qiu and G. Sterman, Phys. Rev. D49, 4493 (1994).

[7] J. W. Qiu and I. Vitev, Phys. Rev. Lett. 93, 262301 (2004).

[8] A. Airapetian et al. [HERMES Collaboration], Eur. Phys. J. C 20, 479 (2001).

[9] A. Accardi, in this proceedings.

[10] X. F. Guo and X. N. Wang, Phys. Rev. Lett. 85, 3591 (2000); X. N. Wang and X. F. Guo, Nucl. Phys. A 696, 788 (2001); E. Wang and X. N. Wang, Phys. Rev. Lett. 89, 162301 (2002). 